\title{
Estimation of a fold convolution in additive noise model with compactly supported noise density
}

\author{
Cao Xuan Phuong
}

\begin{abstract}
Consider the model $Y=X+Z$, where $Y$ is an observable random variable, $X$ is an unobservable random variable with unknown density $f$, and $Z$ is a random noise independent of $X$. The density $g$ of $Z$ is known exactly and assumed to be compactly supported. We are interested in estimating the $m$ - fold convolution $f_{m}=f * \ldots * f$ on the basis of independent and identically distributed (i.i.d.) observations $Y_{1}, \ldots, Y_{n}$ drawn from the distribution of $Y$. Based on the observations as well as the ridge-parameter regularization method, we propose an estimator for the function $f_{m}$ depending on two regularization parameters in which a parameter is given and a parameter must be chosen. The proposed estimator is shown to be consistent with respect to the mean integrated squared error under some conditions of the parameters. After that we derive a convergence rate of the estimator under some additional regular assumptions for the density $f$.
\end{abstract}

Index Terms - estimator, compactly supported noise density, convergence rate

\section{INTRODUCTION}

$I^{n}$ $\mathrm{n}$ this paper, we consider the additive noise model

$$
Y=X+Z
$$

where $Y$ is an observable random variable, $X$ is an unobservable random variable with unknown density $f$, and $Z$ is an unobservable random noise with known density $g$. The density $g$ is called noise density. We also suppose that $X$ and $Z$ are independent. Estimating $f$ on basis of i.i.d.

Received 06-05-2017; Accepted 15-05-2017; Published 108-2018

Author: Cao Xuan Phuong- Ton Duc Thang University (xphuongcao@gmail.com) observations of $Y$ has been known as the density deconvolution problem in statistics. This problem has received much attention during two last decades. Various estimation techniques for $f$ can be found in Carroll-Hall [1], Stefanski-Carroll [2], Fan [3], Neumann [4], Pensky-Vidakovic [5], Hall-Meister [6], Butucea-Tsybakov [7], Johannes [8], among others.

This problem has concerned with many real-life problems in econometrics, biometrics, signal reconstruction, etc. For example, when an input signal passes through a filter, output signal is usually disturbed by an additional noise, in which the output signal is observable, but the input signal is not.

Let $Y_{1}, \ldots, Y_{n}$ be $n$ i.i.d. observations of $Y$.

In the present paper, instead of estimating $f$, we focus on the problem of estimating the $m$-fold convolution

$$
f_{m}=\underbrace{f * \ldots * f}_{m \text { times }}, \quad m \in \mathbb{Z}^{+},
$$

based on the observations. In the free-error case, i.e. $Z \equiv 0$, there are many papers related to this problem, such as Frees [9], Saavedra-Cao [10], Ahmad-Fan [11], Ahmad-Mugdadi [12], Chesneau et al. [13], Chesneau-Navarro [14], and references therein. For $m=1$, the problem of estimating $f_{m}$ reduces to the density deconvolution problem. To the best of our knowledge, for $m \in \mathbb{Z}^{+}, m \geq 2$, so far this problem has been only studied by Chesneau et al. [15]. In that paper, the authors constructed a kernel type of estimator for $f_{m}$ under the assumption that $g^{\mathrm{ft}}$ is nonvanishing on $\mathbb{R}$, where the function $g^{\mathrm{ft}}(t)=\int_{\mathbb{R}} f(x) e^{i t x} d t$ is the Fourier transform of $g$. The latter assumption is fulfilled with many usual densities, such as normal, Cauchy, Laplace, gamma, chi-square densities. However, there are also several cases of $g$ that cannot be applied to this paper. For 
instance, the case in which $g$ is a uniform density or a compactly supported density in general. In the present paper, as a continuation of the paper of Chesneau et al. [15], we consider the case of compactly supported noise density $g$. In fact, the problem was studied by Trong-Phuong [16] in the case of $m=1$; however, the problem has more challenge with $m \in \mathbb{Z}^{+}, m \geq 2$.

The rest of our paper consists of three sections. In Section 2, we establish our estimator. In Section 3 , we state main results of our paper. Finally, some conclusions are presented in Section 4.

For convenience, we introduce some notations. For two sequences $\left(u_{n}\right)$ and $\left(v_{n}\right)$ of positive real numbers, we write $u_{n}=O\left(v_{n}\right)$ if the sequence $\left(u_{n} / v_{n}\right)$ is bounded. The number of $k$ combinations from a set of $p$ elements is denoted by $C_{p}^{k}$. The number $\lambda(A)$ is the Lebesgue measure of a set $A \subset \mathbb{R}$. For a function $\varphi \in L^{p}(\mathbb{R}), \quad 1 \leq p \leq \infty, \quad$ the symbol $\|\varphi\|_{p}$ represents the usual $L^{p}(\mathbb{R})$-norm of $\varphi$. For a function $\quad \phi: \mathbb{R} \rightarrow \mathbb{C}, \quad$ we define $Z(\phi)=\{x \in \mathbb{R}: \phi(x)=0\} \quad$ and $\operatorname{supp}(\phi)=\overline{\mathbb{R} \backslash Z(\phi)}$, the closure in $\mathbb{R}$ of the set $\mathbb{R} \backslash Z(\phi)$. Regarding the Fourier transform, we recall that $\left(\phi^{\mathrm{ft}}\right)^{\mathrm{ft}}(x)=2 \pi \phi(-x)$ for $x \in \mathbb{R}$, $\phi \in L^{1}(\mathbb{R}) \cap L^{2}(\mathbb{R}), \quad$ and moreover, $\left\|\phi^{\mathrm{ft}}\right\|_{2}=\sqrt{2 \pi}\|\phi\|_{2}$, which is called the Parseval identity.

\section{METHODS}

We now describe the method for constructing an estimator for $f_{m}$. First, from the equation (2) we have $f_{m}^{\mathrm{ft}}(t)=\left[f^{\mathrm{ft}}(t)\right]^{m}$. Also, from the independence of $X$ and $Z$, we obtain $h=f * g$, where $h$ is density of $Y$. The latter equation gives $h^{\mathrm{ft}}(t)=f^{\mathrm{ft}}(t) \cdot g^{\mathrm{ft}}(t)$, so $f_{m}^{\mathrm{ft}}(t)=\left[h^{\mathrm{ft}}(t) / g^{\mathrm{ft}}(t)\right]^{m}$ if $g^{\mathrm{ft}}(t) \neq 0$. Then applying the Fourier inversion formula, we can obtain an estimator for $f_{m}$. However, it is very dangerous to use $\left[h^{\mathrm{ft}}(t) / g^{\mathrm{ft}}(t)\right]^{m}$ as an estimator for $f_{m}^{\mathrm{ft}}(t)$ in case $g^{\mathrm{ft}}$ can vanish on $\mathbb{R}$. In this case, to avoid division by numbers very close to zero, $1 / g^{\mathrm{ft}}(t)$ is replaced by the quantity $r(t)=g^{\mathrm{ft}}(-t) / \max \left\{\left|g^{\mathrm{ft}}(t)\right|^{2} ; \delta|t|^{a}\right\}, \quad$ called the ridge function. Here $a>1 / m$ is a given parameter, and $\delta>0$ is a regularization parameter that will be chosen according to $n$ later so that $\delta \rightarrow 0$ as $n \rightarrow \infty$. We then obtain an estimator for $f_{m}^{\mathrm{ft}}(t) \quad$ in the form $\Phi(t)=\left[r(t) h^{\mathrm{ft}}(t)\right]^{m}$. Nevertheless, the function $\Phi(t)$ depends on the Fourier transform $h^{\mathrm{ft}}(t)$, which is an unknown quantity, and so, we cannot use $\Phi(t)$ to estimate $f_{m}^{\mathrm{ft}}(t)$. Fortunately, from the i.i.d. observations $Y_{1}, \ldots, Y_{n}$, we can estimate $h^{\mathrm{ft}}(t)$ by the empirical characteristics function $\hat{h}^{\mathrm{ft}}(t)=n^{-1} \sum_{j=1}^{n} e^{i t Y_{j}}$. Hence, another estimator for $f_{m}^{\mathrm{ft}}(t)$ is proposed by $\Phi(t)=\left[r(t) \hat{h}^{\mathrm{tt}}(t)\right]^{m}$. Finally, using the Fourier inversion formula, we derive an estimator for $f_{m}$ in the final form

$$
\begin{aligned}
& \hat{f}_{m, \delta}(x):=\frac{1}{2 \pi} \int_{\mathbb{R}} e^{-i t x} \Phi(t) d t \\
& =\frac{1}{2 \pi} \int_{\mathbb{R}} e^{-i t x}\left(\frac{g^{\mathrm{ft}}(-t) \hat{h}^{\mathrm{ft}}(t)}{\max \left\{\left|g^{\mathrm{ft}}(t)\right|^{2} ; \delta|t|^{a}\right\}}\right)^{m} d t .
\end{aligned}
$$

Note that the condition $a>1 / m$ implies $\Phi \in L^{1}(\mathbb{R}) \cap L^{2}(\mathbb{R})$ almost surely. Thus, the estimator $\hat{f}_{m, \delta}(x)$ is well-defined for all values of $x \in \mathbb{R}$, and moreover, $\hat{f}_{m, \delta}$ belongs to $L^{2}(\mathbb{R})$.

\section{RESULTS}

In this section, we consider consistency and convergence rate of the estimator $\hat{f}_{m, \delta}$ given in (3) under the mean integrated squared error $\operatorname{MISE}\left(\hat{f}_{m, \delta}, f_{m}\right)=\mathbb{E}\left\|\hat{f}_{m, \delta}-f_{m}\right\|_{2}^{2}$. First, a general bound for $\operatorname{MISE}\left(\hat{f}_{m, \delta}, f_{m}\right)$ is given in the following proposition.

Proposition 1. Let $\hat{f}_{m, \delta}, m \geq 1$, be as in (3) with $a>1 / m$ and $0<\delta<1$. Suppose that $f \in L^{2}(\mathbb{R})$. Then we have 
$\operatorname{MISE}\left(\hat{f}_{m, \delta}, f_{m}\right) \leq\left.\frac{1}{\pi} \int_{\mathbb{R}}\left|\frac{\left|g^{\mathrm{ft}}(t)\right|^{2 m}}{\max \left\{\left|g^{\mathrm{ft}}(t)\right|^{2} ; \delta|t|^{a}\right\}^{m}}-1\right| f^{\mathrm{ft}}(t)\right|^{2 m} d t$
$+\frac{2^{m}-1}{\pi} \sum_{k=1}^{m} C_{m}^{k} \frac{C_{k}}{n^{k}} \int_{\mathbb{R}} \frac{\left|g^{\mathrm{ft}}(t)\right|^{4 m-2 k}\left|f^{\mathrm{ft}}(t)\right|^{2(m-k)}}{\max \left\{\left|g^{\mathrm{ft}}(t)\right|^{2} ; \delta|t|^{a}\right\}^{2 m}} d t$,

where $C_{k}=(72 k)^{2 k}(2 k /(2 k-1))^{k}, k=1, \ldots, m$.

Proof. Since $f$ is a density and is in $L^{2}(\mathbb{R})$, we deduce $f_{m} \in L^{1}(\mathbb{R}) \cap L^{2}(\mathbb{R})$, so $f_{m}^{\mathrm{ft}} \in L^{2}(\mathbb{R})$. Using the Parseval identity, the Fubini theorem and the binomial theorem, we obtain

$\operatorname{MISE}\left(\hat{f}_{m, \delta}, f_{m}\right)=\frac{1}{2 \pi} \int_{\mathbb{R}} \mathbb{E}\left|\hat{f}_{m, \delta}^{\mathrm{n}}(t)-f_{m}^{\mathrm{n}}(t)\right|^{2} d t$

$\left.=\frac{1}{2 \pi} \int \mathbb{E} \mathbb{\mathbb { R }}\left(\frac{g^{\mathrm{n}}(-t) \hat{h}^{\mathrm{n}}(t)}{\max \left\{\left|g^{\mathrm{n}}(t)\right|^{2} ; \delta t^{\mathrm{a}}\right\}}\right\}\right)^{m}-\left.\left[f^{\mathrm{n}}(t)\right]^{m}\right|^{2} d t$

$=\frac{1}{2 \pi} \int \mathbb{E} \mathbb{E}\left(\frac{g^{\mathrm{n}}(-t)}{\max \left\{\left.g^{\mathrm{n}}(t)\right|^{2} ; \delta\left|t^{\mathrm{a}}\right|^{\mathrm{a}}\right\}}\left[\hat{h}^{\mathrm{n}}(t)-h^{\mathrm{n}}(t)+h^{\mathrm{n}}(t)\right]\right)^{m}-\left.\left[f^{\mathrm{n}}(t)\right]^{m}\right|^{2} d t$

$\left.=\frac{1}{2 \pi} \int_{\mathbb{R}} \mathbb{E} \mid \frac{g^{\mathrm{n}}(-t)}{\max \left\{\left|g^{\mathrm{n}}(t)\right|^{2} ; \delta\left|t^{\mathrm{a}}\right|\right\}}\right)^{m} \sum_{k=0}^{m} C_{m}^{k}\left[\hat{h}^{\mathrm{n}}(t)-h^{\mathrm{n}}(t)\right]^{k}\left[h^{\mathrm{n}}(t)\right]^{m-k}-\left.\left[f^{\mathrm{n}}(t)\right]^{m}\right|^{2} d t$.

Using the inequality $\left|z_{1}+z_{2}\right|^{2} \leq 2\left|z_{1}\right|^{2}+2\left|z_{2}\right|^{2}$ with $\left(z_{1}, z_{2}\right) \in \mathbb{C}^{2}$ yields

$$
\operatorname{MiSE}\left(\hat{f}_{m, \delta}, f_{m}\right) \leq \frac{1}{\pi}\left(I_{1}+I_{2}\right),
$$

where

$$
\begin{gathered}
I_{1}=\int_{\mathbb{R}}\left|\left(\frac{g^{\mathrm{ft}}(-t)}{\max \left\{\left|g^{\mathrm{ft}}(t)\right|^{2} ; \delta|t|^{a}\right\}}\right)^{m}\left[h^{\mathrm{ft}}(t)\right]^{m}-\left[f^{\mathrm{ft}}(t)\right]^{m}\right|^{2} d t, \\
I_{2}=\int_{\mathbb{R}} \mathbb{E}\left|\left(\frac{g^{\mathrm{ft}}(-t)}{\max \left\{\left|g^{\mathrm{ft}}(t)\right|^{2} ; \delta||^{a}\right\}}\right)^{m} \sum_{k=1}^{m} C_{m}^{k}\left[h^{\mathrm{t}}(t)-h^{\mathrm{ft}}(t)\right]^{k}\left[h^{\mathrm{t}}(t)\right]^{m-k}\right|^{2} d t .
\end{gathered}
$$

Since $h^{\mathrm{ft}}(t)=f^{\mathrm{ft}}(t) \cdot g^{\mathrm{ft}}(t)$ and $g^{\mathrm{ft}}(-t)=\overline{g^{\mathrm{ft}}(t)}$, in which $\overline{g^{\mathrm{ft}}(t)}$ denotes the conjugate of $g^{\mathrm{ft}}(t)$, we have

$$
\begin{aligned}
I_{1} & =\int_{\mathbb{R}}\left|\left(\frac{\left|g^{\mathrm{ft}}(t)\right|^{2} f^{\mathrm{ft}}(t)}{\max \left\{\left|g^{\mathrm{ft}}(t)\right|^{2} ; \delta|t|^{a}\right\}}\right)^{m}-\left[f^{\mathrm{ft}}(t)\right]^{m}\right|^{2} d t \\
& =\int_{\mathbb{R}}\left|\frac{\left|g^{\mathrm{ft}}(t)\right|^{2 m}}{\max \left\{\left|g^{\mathrm{ft}}(t)\right|^{2} ; \delta\left|t^{a}\right|^{m}\right.}-1\right|\left|f^{\mathrm{ft}}(t)\right|^{2 m} d t,
\end{aligned}
$$

$I_{2} \leq \int_{\mathbb{R}} \frac{\left|g^{\mathrm{ft}}(t)\right|^{2 m}}{\max \left\{\left|g^{\mathrm{ft}}(t)\right|^{2} ;\left.\delta|t|\right|^{a}\right\}^{2 m}} \mathbb{E}\left(\sum_{k=1}^{m} C_{m}^{k}\left|\hat{h}^{\mathrm{n}}(t)-h^{\mathrm{ft}}(t)\right|^{k}\left|h^{\mathrm{f}}(t)\right|^{m-k}\right)^{2} d t$

$\leq \int_{\mathbb{R}} \frac{\left|g^{\mathrm{t}}(t)\right|^{2 m}}{\max \left\{\left|g^{\mathrm{ft}}(t)\right|^{2} ; \delta|t|^{a}\right\}^{2 m}} \sum_{k=1}^{m} C_{m}^{k} \cdot \sum_{k=1}^{m} C_{m}^{k} \mathbb{E}\left[\left|\hat{h}^{\mathrm{t}}(t)-h^{\mathrm{ft}}(t)\right|^{2 k}\right]\left|h^{\mathrm{ft}}(t)\right|^{2(m-k)} d t$
$\left.=\left(2^{m}-1\right) \sum_{k=1}^{m} C_{m}^{k} \int_{\mathbb{R}} \frac{\left|g^{\mathrm{ft}}(t)\right|^{4 m-2 k}\left|f^{\mathrm{ft}}(t)\right|^{2(m-k)}}{\max \left\{\left|g^{\mathrm{ft}}(t)\right|^{2} ; \delta|t|^{a}\right\}^{2 m}} \mathbb{E}\left[\mid \sum_{j=1}^{n}-\frac{1}{n}\left[e^{i Y_{j}}-\mathbb{E}\left(e^{i Y_{j}}\right)\right]\right]^{2 k}\right] d t$.

Define $\quad U_{j}=n^{-1}\left[e^{i t Y_{j}}-\mathbb{E}\left(e^{i t Y_{j}}\right)\right], \quad j=1, \ldots, n$.

Clearly, the sequence $\left(U_{j}\right)_{j=1, \ldots, n}$ satisfies the conditions of Lemma A.1 in Chesneau et al. [15], and moreover, $\left|U_{j}\right| \leq 2 / n$. Hence, applying Lemma A.1 in Chesneau et al. [15] with $p=2 k>1$, we get

$$
\begin{aligned}
& \mathbb{E}\left[\left|\sum_{j=1}^{n} U_{j}\right|^{2 k}\right] \leq(36 k)^{2 k}\left(\frac{2 k}{2 k-1}\right)^{k} \mathbb{E}\left[\left.\left.\left|\sum_{j=1}^{n}\right| U_{j}\right|^{2}\right|^{k}\right] \\
& \leq(36 k)^{2 k}\left(\frac{2 k}{2 k-1}\right)^{k}\left(\frac{4}{n}\right)^{k}=(72 k)^{2 k}\left(\frac{2 k}{2 k-1}\right)^{k} \cdot \frac{1}{n^{k}}=: C_{k} \frac{1}{n^{k}} .
\end{aligned}
$$

Thus,

$$
I_{2} \leq\left(2^{m}-1\right) \sum_{k=1}^{m} C_{m}^{k} \frac{C_{k}}{n^{k}} \int_{\mathbb{R}} \frac{\left|g^{\mathrm{ft}}(t)\right|^{4 m-2 k}\left|f^{\mathrm{ft}}(t)\right|^{2(m-k)}}{\max \left\{\left|g^{\mathrm{ft}}(t)\right|^{2} ; \delta|t|^{a}\right\}^{2 m}} d t .
$$

From (4) - (6), we obtain the desired conclusion.

Proposition 2. Let the assumptions of Proposition 1 hold. Then there exists a $k_{0}>0$ depending only on $g$ such that 


$$
\begin{aligned}
& \sum_{k=1}^{m} C_{m}^{k} \frac{C_{k}}{n^{k}} \int_{\mathbb{R}} \frac{\left|g^{\mathrm{ft}}(t)\right|^{4 m-2 k}\left|f^{\mathrm{ft}}(t)\right|^{2(m-k)}}{\max \left\{\left|g^{\mathrm{ft}}(t)\right|^{2} ; \delta|t|^{a}\right\}^{2 m}} d t \\
& \leq\left(2^{2 m+1} k_{0}+\int_{|t|>k_{0}} \frac{1}{|t|^{m a}} d t\right)\left(\sum_{k=1}^{m} C_{m}^{k} C_{k}\right) \frac{1}{n \delta^{m}} .
\end{aligned}
$$

Proof. Since the function $\left|g^{\mathrm{ft}}\right|$ is continuous on $\mathbb{R}$ and $\left|g^{\mathrm{ft}}(0)\right|=1$, there is a constant $k_{0}>0$ depending only on $g$ such that $\left|g^{\mathrm{ft}}(t)\right| \geq 1 / 2$ for all $|t| \leq k_{0}$. Then for $k=1, \ldots, m$ we have

$$
\begin{aligned}
& \int_{\mathbb{R}} \frac{\left|g^{\mathrm{ft}}(t)\right|^{4 m-2 k}}{\max \left\{\left|g^{\mathrm{ft}}(t)\right|^{2} ; \delta|t|^{a}\right\}^{2 m}}\left|f^{\mathrm{ft}}(t)\right|^{2(m-k)} d t \\
& =\int_{\mathbb{R}} \frac{\left|g^{\mathrm{ft}}(t)\right|^{2 m}}{\max \left\{\left|g^{\mathrm{ft}}(t)\right|^{2} ; \delta|t|^{a}\right\}^{2 m}}\left|h^{\mathrm{ft}}(t)\right|^{2(m-k)} d t \\
& \leq \int_{|| \mid \leq k_{0}} \frac{1}{\left|g^{\mathrm{ft}}(t)\right|^{2 m}} d t+\int_{|| \mid k_{0}} \frac{1}{\max \left\{\left|g^{\mathrm{ft}}(t)\right|^{2} ; \delta|t|^{a}\right\}^{m}}\left|h^{\mathrm{ft}}(t)\right|^{2(m-k)} d t \\
& \leq\left(2^{2 m+1} k_{0}+\int_{|| l>k_{0}} \frac{1}{|t|^{m a}} d t\right) \frac{1}{\delta^{m}} .
\end{aligned}
$$

Hence,

$$
\begin{aligned}
& \sum_{k=1}^{m} C_{m}^{k} \frac{C_{k}}{n^{k}} \int_{\mathbb{R}} \frac{\left|g^{\mathrm{ft}}(t)\right|^{4 m-2 k}\left|f^{\mathrm{ft}}(t)\right|^{2(m-k)}}{\max \left\{\left|g^{\mathrm{ft}}(t)\right|^{2} ; \delta|t|^{a}\right\}^{2 m}} d t \\
& \leq\left(2^{2 m+1} k_{0}+\int_{|t|>k_{0}} \frac{1}{|t|^{m a}} d t\right) \frac{1}{\delta^{m}} \sum_{k=1}^{m} C_{m}^{k} \frac{C_{k}}{n^{k}} \\
& \leq\left(2^{2 m+1} k_{0}+\int_{|t|>k_{0}} \frac{1}{|t|^{m a}} d t\right)\left(\sum_{k=1}^{m} C_{m}^{k} C_{k}\right) \frac{1}{n \delta^{m}} .
\end{aligned}
$$

The proof of the proposition is completed.

The mean consistency of the estimator $\hat{f}_{m, \delta}$ is given in the following theorem.

Theorem 3. Suppose that $f \in L^{2}(\mathbb{R})$ and $\lambda\left(Z\left(g^{\mathrm{ft}}\right)\right)=0$. Let $\hat{f}_{m, \delta}$ be as in (3), where $a>1 / m$ and $\delta$ is a positive parameter depending on $n$ such that $\delta \rightarrow 0$ and $n \delta^{m} \rightarrow \infty$ as $n \rightarrow \infty$. Then $\operatorname{MISE}\left(\hat{f}_{m, \delta}, f_{m}\right) \rightarrow 0$ as $n \rightarrow \infty$.
Proof. Since $\lambda\left(Z\left(g^{\mathrm{ft}}\right)\right)=0$ and the Lebesgue dominated convergence theorem, we get

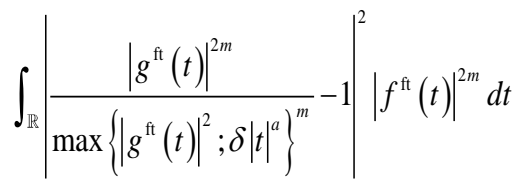

$$
=\left.\int_{\mathbb{R} \backslash Z\left(g^{\mathrm{ft}}\right)}\left|\frac{\left|g^{\mathrm{ft}}(t)\right|^{2 m}}{\max \left\{\left|g^{\mathrm{ft}}(t)\right|^{2} ; \delta|t|^{a}\right\}^{m}}-1\right| f^{\mathrm{ft}}(t)\right|^{2 m} d t
$$

$\rightarrow 0$ as $n \rightarrow \infty$.

Combining this with Proposition 1, Proposition 2 and the assumptions of the present theorem, we obtain the conclusion.

Remark 4. The condition $\lambda\left(Z\left(g^{\mathrm{ft}}\right)\right)=0$ in Theorem 3 is satisfied for normal, gamma, Cauchy, Laplace, uniform, triangular densities, among others. In particular, if the noise density $g$ is a compactly supported, the Fourier transform $g^{\mathrm{ft}}$ can be extended to an analytic function on $\mathbb{C}$. This implies the set $Z\left(g^{\mathrm{ft}}\right)$ is at most countable, so $\lambda\left(Z\left(g^{\mathrm{ft}}\right)\right)=0$.

In the rest of this section, we study rates of convergence of $\operatorname{MISE}\left(\hat{f}_{m, \delta}, f_{m}\right)$. To do this, we need prior information for $f$ and $g$. Concerning the density $f$, we assume that it belongs to the class

$$
\begin{array}{r}
\mathscr{F}(\alpha, L)=\left\{\text { density } \phi \text { on } \mathbb{R}: \int_{\mathbb{R}}\left|\phi^{\mathrm{ft}}(t)\right|^{2}\left(1+t^{2}\right)^{\alpha} d t \leq L,\right. \\
\left.\sup _{u \in \mathbb{R}}\left|\phi^{\mathrm{tt}}(u)\right|^{2}\left(1+u^{2}\right)^{\alpha} \leq L\right\}
\end{array}
$$

with $\alpha>1 / 2, L>0$. The class $\mathscr{F}(\alpha, L)$ contains many important densities, for example, normal and Cauchy densities. Note that $\mathscr{F}(\alpha, L) \subset L^{1}(\mathbb{R}) \cap L^{2}(\mathbb{R})$. In fact, for positive integer $\alpha$, if a density $\phi$ is in $L^{2}(\mathbb{R})$ having weak derivatives $\phi^{(l)}, l=1, \ldots, \alpha$, and the weak derivatives are also in $L^{2}(\mathbb{R})$, then $\phi$ belongs to $\mathscr{F}(\alpha, L)$ for $L>0$ large enough. Regarding the noise density $g$, we consider the following classes of $g$ : 
$\mathscr{F}(M)=\left\{\right.$ density $\phi$ on $\mathbb{R}: \phi \in L^{2}(\mathbb{R})$,

$$
\operatorname{supp}(\phi) \subset[-M, M]\},
$$

$\mathscr{F}\left(c_{1}, c_{2}, d, \beta\right)$

$=\left\{\right.$ density $\phi$ on $\left.\mathbb{R}: c_{1} e^{-d||^{\beta}} \leq\left|\phi^{\mathrm{tt}}(t)\right| \leq c_{2} e^{-d||^{\beta}}, \forall t \in \mathbb{R}\right\}$,

The class $\mathscr{F}(M)$ includes compactly supported densities on $[-M, M]$. The class $\mathscr{F}\left(c_{1}, c_{2}, d, \beta\right)$ contains densities in which Fourier transforms converge to zero with exponential rate of order $\beta$. Normal and Cauchy densities are typical examples of $\mathscr{F}\left(c_{1}, c_{2}, d, \beta\right)$. In fact, using the Fourier inversion formula and the Lebesgue dominated convergence theorem, one can show that each element of $\mathscr{F}\left(c_{1}, c_{2}, d, \beta\right)$ is an infinitely differentiable function on $\mathbb{R}$. Hence, $\mathscr{F}\left(c_{1}, c_{2}, d, \beta\right)$ is often called the class of "supersmooth" densities. In fact, the case of $g \in \mathscr{F}\left(c_{1}, c_{2}, d, \beta\right)$ has been studied in Chesneau et al. [15]. The reason for considering this class in the present paper is that we want to demonstrate that the estimator $\hat{f}_{m, \delta}$ can also be attained the convergence rate established in Chesneau et al. [15].

Now, we consider the case $g \in \mathscr{F}(M)$. Before stating main result of our paper, we need the following auxiliary lemma. This auxiliary lemma is not a new result. It is quite similar to Theorem 3 in Trong-Phuong [16].

Lemma 5. Suppose $g \in \mathscr{F}(M)$. Given $\mu>1$. For $\rho>0$ small enough, we choose an $R>0$ depending on $\rho$ such that $2 e M R\left[(\mu+1) \ln R+\ln \left(15 e^{3}\right)\right]=\ln \left(\rho^{-1}\right)$. Then for $\rho>0$ small enough we have in which $M, c_{1}, c_{2}, d, \beta$ are positive constants.

Theorem 6. Let $\alpha>1 / 2, L>0$. Assume that $g \in \mathscr{F}(M)$ with $M>0$. Let $\hat{f}_{m, \delta}$ be as in (3) for a known $a>1 / m$ and $\delta=n^{-\tau}$ with $0<\tau<1 / m$. Then we have $\sup _{f \in \mathscr{F}(\alpha, L)} \operatorname{MISE}\left(\hat{f}_{m, \delta}, f_{m}\right)=O\left\{(\ln n)^{-m \alpha}\right\}$.

Proof. Suppose $f \in \mathscr{F}(\alpha, L)$. We take $\mu=2 m \alpha>1, \quad 0<\tau<1 / m$ and $\rho=n^{-v}$ with $0<v<\tau / 2$. Then applying Lemma 5 gives that there exists an $R>0$ depending on $n$ such that $\left[30(2 m \alpha+1) M e^{4}\right]^{-1 / 2}\left[\ln \left(n^{v}\right)\right]^{1 / 2} \leq R \leq(2 e M)^{-1} \ln \left(n^{v}\right)$ , and $\lambda\left(B_{R, \rho}\right) \leq 2 R^{-2 m \alpha}$ for $n$ large enough. Now, for $0<\delta<R^{-a} \rho^{2}$, we have

$$
\begin{aligned}
& J:=\int_{\mathbb{R}}\left|\frac{\left|g^{\mathrm{ft}}(t)\right|^{2 m}}{\max \left\{\left|g^{\mathrm{ft}}(t)\right|^{2} ; \delta|t|^{a}\right\}^{m}}-1\right|\left|f^{\mathrm{ft}}(t)\right|^{2 m} d t \\
& \leq \int_{\left|s^{\mathrm{n}}(t)\right|<\sqrt{\left.\delta \delta\right|^{a}}}\left|f^{\mathrm{ft}}(t)\right|^{2 m} d t \\
& =\int_{|t| \leq R,\left|g^{\mathrm{n}}(t)\right| \leq \rho}\left|f^{\mathrm{ft}}(t)\right|^{2 m} d t+\int_{|||\leq R, \rho<| g^{\mathrm{n}}(t) \mid<\sqrt{\delta|t|^{a}}}\left|f^{\mathrm{ft}}(t)\right|^{2 m} d t \\
& +\int_{|t|>R,\left|g^{\mathrm{ft}}(t)\right|<\sqrt{\delta \mid t^{|l|}}}\left|f^{\mathrm{ft}}(t)\right|^{2 m} d t \\
& \leq \lambda\left(B_{R, \rho}\right)+\int_{|t|>R}\left|f^{\mathrm{ft}}(t)\right|^{2 m} d t \leq 2 R^{-2 m \alpha}+\int_{|t|>R}\left|f^{\mathrm{ft}}(t)\right|^{2 m} d t,
\end{aligned}
$$

where we note that $\left\{t \in \mathbb{R}:|t| \leq R, \rho<\left|g^{\mathrm{ft}}(t)\right|<\sqrt{\delta|t|^{a}}\right\}=\varnothing$.

Moreover, since $f \in \mathscr{F}(\alpha, L)$, we derive

$\int_{|t|>R}\left|f^{\mathrm{ft}}(t)\right|^{2 m} d t$

$=\int_{|| \mid>R}\left|f^{\mathrm{ft}}(t)\right|^{2}\left(1+t^{2}\right)^{\alpha}\left[\left|f^{\mathrm{ft}}(t)\right|^{2}\left(1+t^{2}\right)^{\alpha}\right]^{m-1}\left(1+t^{2}\right)^{-m \alpha} d t$ $\leq L^{m} R^{-2 m \alpha}$.

Hence,

$$
\begin{aligned}
& J \leq\left(2+L^{m}\right) R^{-2 m \alpha} \\
& \leq\left(2+L^{m}\right)\left[30(2 m \alpha+1) M e^{4}\right]^{m \alpha}\left[\ln \left(n^{v}\right)\right]^{-m \alpha}(7) \\
& =O\left\{(\ln n)^{-m \alpha}\right\} .
\end{aligned}
$$

In addition, we have $\lambda\left(B_{R, \rho}\right) \leq 2 R^{-\mu}$, where $B_{R, \rho}=\left\{t \in \mathbb{R}:|t| \leq R,\left|g^{\mathrm{ft}}(t)\right| \leq \rho\right\}$. theorem.

Main result of our paper is the following 2, $\operatorname{MISE}\left(\hat{f}_{m, \delta}, f_{m}\right)=O\left\{(\ln n)^{-m \alpha}+\left(n \delta^{m}\right)^{-1}\right\}$. Now, we need to choose $\delta>0$ according to $n$ so that $\delta<R^{-a} \rho^{2}$, and rate of convergence of $\left(n \delta^{m}\right)^{-1}$ is faster than that of $(\ln n)^{-m \alpha}$. A possible choice is 
$\delta=n^{-\tau}$. Then the conclusion of the theorem is followed.

Remark 7. The parameter $\delta$ in Theorem 6 does not depend on $\alpha$, the prior degree of smoothness of $f$. Therefore, the estimator $\hat{f}_{m, \delta}(x)$ can be computed with out any knowledge concerning the degree of smoothness.

Finally, we consider the case $g \in \mathscr{F}\left(c_{1}, c_{2}, d, \beta\right)$. We have

Theorem 8. Let $\alpha>1 / 2, L>0$. Suppose that $g \in \mathscr{F}\left(c_{1}, c_{2}, d, \beta\right)$, where $c_{1}, c_{2}, d, \beta$ are the given positive constants. Let $\hat{f}_{m, \delta}$ be as in (3) for a known $a>1 / m$ and $\delta=n^{(1-8 m))\left(16 m^{2}\right)}(\ln n)^{(1-4 a m) /(4 m \beta)}$. Then we have $\sup _{f \in \mathscr{F}(\alpha, L)} \operatorname{MISE}\left(\hat{f}_{m, \delta}, f_{m}\right)=O\left\{(\ln n)^{-2 m \alpha / \beta}\right\}$.

Proof. Suppose $f \in \mathscr{F}(\alpha, L)$. Let $T$ be a positive number that will be selected later. Using the inequality

$0 \leq \max \left\{\left|g^{\mathrm{ft}}(t)\right|^{2} ; \delta|t|^{a}\right\}^{m}-\left|g^{\mathrm{ft}}(t)\right|^{2 m} \leq \delta^{m}|t|^{a m}$ for all $t \in \mathbb{R}$ and the assumptions $g \in \mathscr{F}\left(c_{1}, c_{2}, d, \beta\right)$, $f \in \mathscr{F}(\alpha, L)$, we have

$J:=\int_{\mathbb{R}}\left|\frac{\left|g^{\mathrm{ft}}(t)\right|^{2 m}}{\max \left\{\left|g^{\mathrm{ft}}(t)\right|^{2} ; \delta|t|^{a}\right\}^{m}}-1\right|\left|f^{\mathrm{ft}}(t)\right|^{2 m} d t$ $\leq \int_{\mathbb{R}} \frac{\delta^{2 m}|t|^{2 a m}}{\max \left\{\left|g^{\mathrm{ft}}(t)\right|^{2} ; \delta|t|^{a}\right\}^{2 m}}\left|f^{\mathrm{ft}}(t)\right|^{2 m} d t$

$\leq \int_{|| \mid \leq T} \frac{\delta^{2 m}|t|^{2 a m}}{\left|g^{\mathrm{ft}}(t)\right|^{4 m}}\left|f^{\mathrm{ft}}(t)\right|^{2 m} d t+\int_{|t|>T}\left|f^{\mathrm{ft}}(t)\right|^{2 m} d t$

$\leq\left(c_{1}\right)^{-4 m} \int_{|t| \leq T} \delta^{2 m}|t|^{2 a m} e^{4 m d||^{\beta}}\left|f^{\mathrm{ft}}(t)\right|^{2} d t$

$+\int_{|| \mid T T}\left|f^{\mathrm{ft}}(t)\right|^{2}\left(1+t^{2}\right)^{\alpha}\left[\left|f^{\mathrm{ft}}(t)\right|^{2}\left(1+t^{2}\right)^{\alpha}\right]^{m-1}\left(1+t^{2}\right)^{-m \alpha} d t$ $=O\left\{\delta^{2 m} e^{4 m d T^{\beta}} T^{2 a m}+T^{-2 m \alpha}\right\}$.

Also,
$Q:=\sum_{k=1}^{m} C_{m}^{k} \frac{C_{k}}{n^{k}} \int_{\mathbb{R}} \frac{\left|g^{\mathrm{ft}}(t)\right|^{4 m-2 k}\left|f^{\mathrm{ft}}(t)\right|^{2(m-k)}}{\max \left\{\left|g^{\mathrm{ft}}(t)\right|^{2} ; \delta|t|^{a}\right\}^{2 m}} d t$

$=\frac{C_{m}}{n^{m}} \int_{\mathbb{R}} \frac{\left|g^{\mathrm{ft}}(t)\right|^{2 m}}{\max \left\{\left|g^{\mathrm{ft}}(t)\right|^{2} ; \delta|t|^{a}\right\}^{2 m}} d t$

$+\sum_{k=1}^{m-1} C_{m}^{k} \frac{C_{k}}{n^{k}} \int_{\mathbb{R}} \frac{\left|g^{\mathrm{ft}}(t)\right|^{4 m-2 k}\left|f^{\mathrm{ft}}(t)\right|^{2(m-k)}}{\max \left\{\left|g^{\mathrm{ft}}(t)\right|^{2} ; \delta|t|^{a}\right\}^{2 m}} d t=: Q_{1}+Q_{2}$.

For the quantities $Q_{1}$ and $Q_{2}$, we have the estimates

$$
\begin{aligned}
Q_{1} & \leq \frac{C_{m}}{n^{m}}\left(\int_{|t| \leq T} \frac{1}{\left|g^{\mathrm{ft}}(t)\right|^{2 m}} d t+\int_{|t|>T} \frac{\left|g^{\mathrm{ft}}(t)\right|^{2 m}}{\delta^{2 m}|t|^{2 a m}} d t\right) \\
& =O\left\{\frac{T}{n^{m}} e^{2 m d T^{\beta}}+\frac{1}{n^{m} \delta^{2 m}} T^{1-2 a m} e^{-2 m d T^{\beta}}\right\},
\end{aligned}
$$

$Q_{2} \leq \sum_{k=1}^{m-1} C_{m}^{k} \frac{C_{k}}{n^{k}}\left(\int_{|t| \leq T} \frac{\left|f^{\mathrm{ft}}(t)\right|^{2(m-k)}}{\left|g^{\mathrm{ft}}(t)\right|^{2 k}} d t\right.$

$\left.+\int_{|t|>T} \frac{\left|g^{\mathrm{ft}}(t)\right|^{4 m-2 k}\left|f^{\mathrm{ft}}(t)\right|^{2(m-k)}}{\delta^{2 m}|t|^{2 a m}} d t\right)$

$\leq \sum_{k=1}^{m-1} C_{m}^{k} \frac{C_{k}}{n^{k}}\left(\left(c_{1}\right)^{-2 k} \int_{|t| \leq T} e^{2 k d|t|^{\beta}}\left|f^{\mathrm{ft}}(t)\right|^{2(m-k)} d t\right.$ $\left.+\frac{\left(c_{2}\right)^{4 m-2 k}}{\delta^{2 m}} \int_{|t|>T} e^{-(4 m-2 k) d \mid t^{\beta}} \frac{\left|f^{\mathrm{ft}}(t)\right|^{2(m-k)}}{|t|^{2 a m}} d t\right)$

$\leq \sum_{k=1}^{m-1} C_{m}^{k} \frac{C_{k}}{n^{k}}\left(L\left(c_{1}\right)^{-2 k} e^{2 k d T^{\beta}}\right.$

$$
\left.+L\left(c_{2}\right)^{4 m-2 k} \frac{1}{\delta^{2 m}} e^{-(4 m-2 k) d T^{\beta}} T^{-2 a m}\right)
$$

$\leq \sum_{k=1}^{m-1} C_{m}^{k} C_{k} L \max \left\{\left(c_{1}\right)^{-2 k} ;\left(c_{2}\right)^{4 m-2 k}\right\}$

$$
\cdot\left(\frac{1}{n^{k}} e^{2 k d T^{\beta}}+\frac{1}{n^{k} \delta^{2 m}} e^{-(4 m-2 k) d T^{\beta}} T^{-2 a m}\right) .
$$

Combining Proposition 1 with the estimates of $J$, $Q_{1}$ and $Q_{2}$, we get

$$
\begin{gathered}
\operatorname{MISE}\left(\hat{f}_{m, \delta}, f_{m}\right)=O\left\{\delta^{2 m} e^{4 m d T^{\beta}} T^{2 a m}+T^{-2 m \alpha}\right. \\
\left.+\frac{T}{n^{m}} e^{2 m d T^{\beta}}+\frac{1}{n^{m} \delta^{2 m}} T^{1-2 a m} e^{-2 m d T^{\beta}}\right\} \\
+\sum_{k=1}^{m-1} C_{k} C_{m}^{k} L \max \left\{\left(c_{1}\right)^{-2 k} ;\left(c_{2}\right)^{4 m-2 k}\right\} \times \\
\times\left(\frac{1}{n^{k}} e^{2 k d T^{\beta}}+\frac{1}{n^{k} \delta^{2 m}} e^{-(4 m-2 k) d T^{\beta}} T^{-2 a m}\right) .
\end{gathered}
$$


Choosing $T=((\ln n) /(8 m d))^{1 / \beta}$ yields

$$
\begin{aligned}
& \operatorname{MISE}\left(\hat{f}_{m, \delta}, f_{m}\right) \\
& =O\left\{n^{1 / 2}(\ln n)^{2 a m / \beta} \delta^{2 m}+(\ln n)^{-2 m \alpha / \beta}\right. \\
& \left.\quad+\frac{1}{n^{m-1 / 4}}(\ln n)^{1 / \beta}+\frac{1}{n^{m+1 / 4} \delta^{2 m}}(\ln n)^{(1-2 a m) / \beta}\right\} \\
& +\sum_{k=1}^{m-1} C_{m}^{k} C_{k} L \max \left\{\left(c_{1}\right)^{-2 k} ;\left(c_{2}\right)^{4 m-2 k}\right\} \times \\
& \quad \times O\left(\frac{1}{n^{k[1-1 /(4 m)]}}+\frac{1}{n^{1 / 2+k[1-1 /(4 m)]} \delta^{2 m}}(\ln n)^{-2 a m / \beta}\right) \\
& =O\left\{n^{1 / 2}(\ln n)^{2 a m / \beta} \delta^{2 m}+(\ln n)^{-2 m \alpha / \beta}\right. \\
& \left.+\frac{1}{n^{m-1 / 4}}(\ln n)^{1 / \beta}+\frac{1}{n^{m+1 / 4} \delta^{2 m}}(\ln n)^{(1-2 a m) / \beta}\right\} \\
& +\sum_{k=1}^{m-1} C_{m}^{k} C_{k} L \max \left\{\left(c_{1}\right)^{-2 k} ;\left(c_{2}\right)^{4 m-2 k}\right\} \times \\
& \quad \times O\left(\frac{1}{n^{1-1 /(4 m)}}+\frac{1}{n^{3 / 2-1 /(4 m)} \delta^{2 m}}(\ln n)^{-2 a m / \beta}\right) \\
& =O\left\{n^{1 / 2}(\ln n)^{2 a m / \beta} \delta^{2 m}+(\ln n)^{-2 m \alpha / \beta}\right. \\
& \left.+\frac{1}{n^{3 / 2-1 /(4 m)}}(\ln n)^{1-2 a m / \beta} \frac{1}{\delta^{2 m}}\right\} .
\end{aligned}
$$

Choosing $\delta=n^{(1-8 m) /\left(16 m^{2}\right)}(\ln n)^{(1-4 a m) /(4 m \beta)}$ implies the desired conclusion.

Remark 9. We see that the convergence rate of $\operatorname{MISE}\left(\hat{f}_{m, \delta}, f_{m}\right)$ uniformly over the class $\mathscr{F}(\alpha, L)$ in Theorem 8 is as same as that of Chesneau et al. [15] when $g \in \mathscr{F}\left(c_{1}, c_{2}, d, \beta\right)$. In particular, when $m=1$, the convergence rate also coincides with the optimal rate of convergence proven in Fan [3].

\section{CONCLUSIONS}

We have considered the problem of nonparametric estimation of the $m$-fold convolution $f_{m}$ in the additive noise model (1), where the noise density $g$ is known and assumed to be compactly supported. An estimator for the function $f_{m}$ has been proposed and proved to be consistent with respect to the mean integrated squared error. Under some regular conditions for the density $f$ of $X$, we derive a convergence rate of the estimator. We also have shown that the estimator attains the same rate as the one of Chesneau et al. [15] if the density $g$ is supersmooth. A possible extension of this work is to study our estimation procedure in the case of unknown noise density $g$. We leave this problem for our future research.

\section{REFERENCES}

[1]. R.J. Carroll, P. Hall, "Optimal rates of convergence for deconvolving a density", Journal of American Statistical Association, vol. 83, pp. 1184-1186, 1988.

[2]. L.A. Stefanski, R.J. Carroll, "Deconvoluting kernel density estimators", Statistics, 21, pp. 169-184, 1990.

[3]. J. Fan, "On the optimal rates of convergence for nonparametric deconvolution problems", The Annals of Statistics, 19, pp. 1257-1272, 1991.

[4]. M.H. Neumann, "On the effect of estimating the error density in nonparametric deconvolution", Journal of Nonparametric Statistics, 7, pp. 307-330, 1997.

[5]. M. Pensky, B. Vidakovic, "Adaptive wavelet estimator for nonparametric density deconvolution", The Annals of Statistics, 27, pp. 2033-2053, 1999.

[6]. P. Hall, A. Meister, "A ridge-parameter approach to deconvolution", The Annals of Statistics, 35, pp. 1535$1558,2007$.

[7]. C. Butucea, A.B. Tsybakov, "Sharp optimality in density deconvolution with dominating bias", Theory Probability and Applications, 51, pp. 24-39, 2008.

[8]. J. Johannes, "Deconvolution with unknown error distribution", The Annals of Statistics, 37, pp. 2301-2323 2009.

[9]. E.W. Frees, "Estimating densities of functions of observations," Journal of the American Statistical Association, 89, pp. 517-525, 1994.

[10]. A. Saavedra, R. Cao, "On the estimation of the marginal density of a moving average process", The Canadian Journal of Statistics, 28, pp.799-815, 2000.

[11]. I.A. Ahmad, Y. Fan, "Optimal bandwidth for kernel density estimator of functions of observations", Statistics \& Probability Letters, 51, pp. 245-251, 2001.

[12]. I.A. Ahmad, A.R. Mugdadi, "Analysis of kernel density estimation of functions of random variables", Journal of Nonparametric Statistics, vol. 15, pp. 579-605, 2003.

[13]. C. Chesneau, F. Comte, F. Navarro, "Fast nonparametric estimation for convolutions of densities", The Canadian Journal of Statistics, vol. 41, pp. 617-636, 2013.

[14]. C. Chesneau, F. Navarro, "On a plug-in wavelet estimator for convolutions of densities", Journal of Statistical Theory and Practice, vol. 8, pp. 653-673, 2014.

[15]. C. Chesneau, F. Comte, G. Mabon, F. Navarro, Estimation of convolution in the model with noise, Journal of Nonparametric Statistics, vol. 27, pp. 286$315,2015$.

[16]. D.D. Trong, C.X. Phuong, Ridge-parameter regularization to deconvolution problem with unknown error distribution, Vietnam Journal of Mathematics, vol. 43, pp. 239-256, 2015. 


\title{
Ước lượng một tự tích chập trong một mô hình cộng nhiễu với hàm mật độ nhiễu có giá compact
}

\author{
Cao Xuân Phương \\ Trường Đại học Tôn Đức Thắng \\ Tác giả liên hệ: xphuongcao@gmail.com
}

Ngày nhận bản thảo: 06-05-2017, ngày chấp nhận đăng: 15-05-2017, ngày đăng: 10-08-2018

Tóm tắt - Bài báo này đề cập mô hình $Y=X+Z$, trong đó $Y$ là một biến ngẫu nhiên quan trắc được, $X$ là một biến ngẫu nhiên không quan trắc được với hàm mật độ $f$ chưa biết, và $Z$ là nhiễu ngẫu nhiên độc lập với $X$. Hàm mật độ $g$ của $Z$ được giả thiết biết chính xác và có giá compact. Bài báo nghiên cứu vấn đề ước lượng phi tham số cho tự tích chập $f_{m}=f * \ldots * f$ ( $m$ lần) trên cơ sở mẫu quan trắc $Y_{1}, \ldots, Y_{n}$ độc lập, cùng phân phối được lấy từ phân phối của $Y$. Dựa trên các quan trắc này cũng như phương pháp chỉnh hóa tham số chóp, một ước lượng cho $f_{m}$ phụ thuộc vào hai tham số chỉnh hóa được đề xuất, trong đó một tham số được cho trước và tham số còn lại sẽ được chọn sau. Ước lượng này được chứng tỏ là vững tương ứng với trung bình sai số tích phân bình phương dưới một sổ điều kiện cho các tham số chỉnh hóa. Sau đó, nghiên cứu tốc độ hội tụ của ước lượng dưới một số giả thiết chính quy bồ sung cho hàm mật độ $f$.

Tù̀ khóa - Uớc lương, hàm mật độ nhiễu có giá compact, tốc độ hội tụ 